\title{
Clinical Trial Independent Data Monitoring Committee Member List
}

National Cancer Institute

\section{Source}

National Cancer Institute. Clinical Trial Independent Data Monitoring Committee Member List. NCl Thesaurus. Code C115532.

A directory of the current membership of the Independent Data Monitoring Committee. 